\title{
4 Planmäßiger individueller Wohnungsbau in Samarkand
}

Individueller Wohnungsbau war in Samarkand, wie anderswo in der Sowjetunion, in Zeiten des Wohnraummangels ein wichtiger Bestandteil bei der Lösung dieses Problems. Die Handhabung seitens der Politik und der Verwaltung war jedoch nicht konstant oder konsequent und durchlief in dem betrachteten Zeitraum mehrere Phasen. Einerseits wurden die Rahmenbedingungen durch den politischen Willen und entsprechende unionsweite oder republikanische Programme und Vorgaben gesetzt. Gleichzeitig übten die Interessen unterschiedlicher lokaler Akteure sowie infrastrukturelle Bedingungen, Zwänge und Entwicklungen vor Ort ebenfalls einen starken Einfluss aus.

\section{Planmäßiger individueller Wohnungsbau in den Händen der (Stadt)politik}

Neben dem akuten Mangel an Wohnraum befand sich Samarkand Anfang der 1950er-Jahre vor der Notwendigkeit der weiteren Industrialisierung und vor umfangreichen Infrastrukturmaßnahmen - also weiterem Wachstum und territorialer Erweiterung. Eine wichtige Voraussetzung dafür war die Verfügbarkeit von unbebautem Land, einer Ressource, über die Samarkand bereits zu Beginn der 50er-Jahre nicht in genügendem Maß verfügte. Zur Lösung dieses Problems wurden etappenweise Territorien der umliegenden Kolchosen in das Stadtgebiet eingemeindet - diese Entscheidungen waren mit großem Konfliktpotential und weitreichenden Folgen verbunden.

Wie bereits geschildert, erfolgte im Jahr $1956^{169}$ eine große Überholung und Aktualisierung des Generalplans von 1938, wodurch dieser an die neuen Bedingungen und Bedürfnisse der Stadt angepasst werden sollte. In dem Plan waren unter anderem auch Flächen für die so genannten „Viertel mit planmäßiger individueller Bebauung“ vorgesehen (kvartaly planovoi individual'noi zastroiki). Die größten von ihnen sind auch heute noch auf Satellitenbildern deutlich zu erkennen.

169 Bei Muminov 1970, Bd. II S. 324, ist die Rede von 1951, allerdings ließ sich dieses Datum nicht durch Archivquellen bestätigen, während das Jahr 1956 in zahlreichen Dokumenten Erwähnung findet.

Ә Open Access. (C) 2021 Marya Petrova, publiziert von De Gruyter. (c))BY-NC-ND Dieses Werk ist lizenziert unter der Creative Commons Attribution-NonCommercial-NoDerivatives 4.0 International Lizenz. https://doi.org/10.1515/9783110669367-004 
Die Praxis der Landzuteilung für individuellen Hausbau gab es in Samarkand auch schon vor den bereits mehrfach erwähnten Gesetzen aus den Jahren 1944 und 1948. So berichtete zum Beispiel eine Hausbesitzerin, dass ihre Eltern das Grundstück bereits 1938 erhalten hatten. ${ }^{170}$ Auch Muminov schreibt von einer wohlwollenden Einstellung gegenüber der individuellen Bautätigkeit in den 1920er- und 30er-Jahren, jedoch mit wenigen konkreten Zahlen, sodass es schwierig ist, das konkrete Ausmaß einzuschätzen. ${ }^{171}$ Nach 1948 wurde die Parzellenverteilung intensiviert, unterstützt durch die Vergabe von Krediten. Bis in die Mitte der 1950er-Jahre hatten die städtischen Behörden jedoch Schwierigkeiten, das geforderte Plansoll zu erfüllen. Es mangelte an Bauland und, bevor der aktualisierte Generalplan vorlag, offenbar auch an einer verlässlichen Planung über die genaue Lage solcher Viertel. Dies lässt sich aus den Entscheidungen des städtischen Ispolkom herleiten, nach denen für die Maßnahme vereinzelte recht kleine Grundstücke von zwei bis drei Hektar an unterschiedlichen Orten der Stadt zusammengekratzt wurden. ${ }^{172}$

In einem anderen Dokument des Ispolkom, von Juni 1956, wird das komplette Fehlen von verfügbarem Bauland festgestellt und beim Gebiets-Ispolkom eine Übereignung von insgesamt 50 Hektar Land von fünf umliegenden Kolchosen (jeweils 10 Hektar) beantragt. ${ }^{173}$ Ob diesem konkreten Antrag stattgegeben wurde, lässt sich nicht verfolgen; ein Flächenzuwachs in diesem geringen Umfang konnte das Problem ohnehin nicht nachhaltig lösen. Entscheidungen zu Übereignungen größerer Flächen lassen sich im betrachteten Zeitraum in den Jahren $1953^{174}$ und $1956^{175}$ finden. Allerdings bedeutete ein entsprechender Beschluss der Stadt noch nicht zwingend die Zustimmung der Kolchosen, deren Leitungen nicht immer gewillt waren, die Anordnungen auszuführen. Ein Beschwerdebrief des Stadt-Ispolkom vom April 1957 kritisiert mehrere Kolchosen, welche den behördlichen Übereignungsbeschluss ignorierten und die bürokratisch notwendigen Schritte dazu verweigert hätten bzw. nur kleine und teilweise bereits bebaute Flächen zur Übergabe anboten. Die Rede ist dabei von insgesamt 252

170 Gespräch mit S.A.

171 Muminov 1970, Bd. II S. 100, 152.

172 SamOGA, f. 1617, op. 1, d. 40, 1. 40.

173 SamOGA, f. 1617, op. 1, d. 62, 1. 72.

174 SamOGA, f. 26, op. 1, d. 1675, 1. 66. Hier handelt es sich um eine Liste von fünf Kolchosen, auf deren Mitgliederversammlungen die Übergabe von insgesamt über 100 Hektar beschlossen wurde. Bei Muminov (1970, Bd. II S. 325) taucht das Jahr 1952 auf, allerdings ließ sich dieses nicht durch Archivquellen bestätigen.

175 SamOGA, f. 1617, op. 1, d. 126, 1. 20-23. 
Hektar, vorgesehen für die Errichtung von 6300 individuellen Häusern. ${ }^{176}$ Vor allem bereits bebautes Land stellte für die Stadt ein großes Problem dar, denn das bedeutete nicht nur weniger Bauland als geplant, sondern brachte häufig auch massive Verstöße gegen die Bauvorhaben und damit gegen den Generalplan ans Licht. Daraus ergaben sich weitreichende Konsequenzen für die zentrale Planung, und der Handlungsspielraum der Stadt wurde erheblich eingeengt.

Die städtische Verwaltung stand unter Druck sowohl von Seiten der Bevölkerung - in Form von Beschwerden und langjährigen, immer länger werdenden Wartelisten - als auch seitens der Parteiinstitutionen, die das Plansoll des Wohnungsbaus (in Quadratmetern ausgedrückt) erfüllt sehen wollten. Dabei fehlten der Verwaltung ganz offensichtlich die Ressourcen und auch die Fähigkeit, die Prozesse effektiv zu organisieren. Deutlich erkennbar wird dies in der Kritik, die sich in den Archiven des Stadt-Ispolkom von Jahr zu Jahr, meist sogar im gleichen Wortlaut, wiederfindet. Angeprangert werden dabei diverse Defizite und Verstöße wie „mangelnde Kontrollen“ und undurchsichtige Dokumentation der zuständigen Behörden. Den Vorwürfen zufolge existierte keine einheitliche Warteliste, was Tricksereien ermöglichte und zur Missachtung der korrekten Reihenfolge der Antragstellung führte. Häufig erfolgte die Zuteilung ohne Überprüfung des tatsächlichen Bedarfs, was es einigen Antragstellern ermöglichte, mehrere Grundstücke zugleich zu bebauen und die Häuser dann zu vermieten oder zu verkaufen. So wurde z.B. auf einer Sitzung des Bezirks-Ispolkom im Juni 1963 der Fall der Familie Kabilov besprochen, der es gelang, durch Anträge und eigenmächtige Aneignung an sechs benachbarte Grundstücke zu gelangen. ${ }^{177}$

In diesem Zusammenhang wird den zuständigen Behörden mangelnde Kontrolle des Bauverlaufs vorgeworfen; kritisiert werden diese auch für die schlechte Versorgung der Bürger mit Baumaterial bzw. dafür, dass spezielle Baumärkte die ohnehin schwer erhältlichen Materialien statt an die Bevölkerung an größere Betriebe verkauften. Hinzu kamen Verzögerungen bei der Parzellierung (meist wegen Fehlens von geeignetem Land), bürokratische Hürden, Zuteilung unter Umgehung des Ispolkoms sowie Probleme mit bereits bebautem Kolchosenland

176 SamOGA, f. 26, op. 1, d. 1972, 1. 19.

177 SamOGA, f. 1658, op. 2, d. 109, 1. 95-97. In diesem Fall wurden Machenschaften aufgedeckt und diverse Konsequenzen bis hin zur Übergabe des Falls an die Staatsanwaltschaft angeordnet. Inwiefern diese letztlich umgesetzt wurden, kann nicht endgültig gesagt werden, da hierfür die Quellen fehlen. 
usw. ${ }^{178}$ Die Kritik erscheint in den Akten sehr routiniert, während viele der Beschlüsse und Anordnungen, die der Gebiets-Ispolkom über die Stadt-Ispolkoms verhängte, eher Appellcharakter haben. ${ }^{179}$

Ein genaues Datum lässt sich nicht feststellen, jedoch indirekt machen die vorhandenen Quellen deutlich, dass es der Stadt im Zeitraum 1956/1957 gelang, einige Territorien von den umliegenden Kolchosen übereignet zu bekommen. Zusammen mit der verbesserten Finanzierung und dem unionsweiten Wohnungsbauprogramm kamen damit in der zweiten Hälfte der 50er-Jahre für eine kurze Zeit mehrere günstige Faktoren zusammen, die zu einem regelrechten Boom an Zuteilungen und individuellem Häuserbau führten. Innerhalb von drei Jahren (1957-1959) wurden planmäßige Eigenheimviertel auf einer Gesamtfläche von 250 Hektar errichtet. Entsprechend hoch war die Zahl der zugeteilten Parzellen: 1015 Stück im Jahr 1957, 2000 im Jahr 1958 und immer noch 950 Parzellen im Jahr 1959. Laut einem Bericht aus dem Jahr 1960 konnte damit eine über fünf Jahre angestaute Warteliste abgearbeitet werden. ${ }^{180}$ Das Problem wurde damit jedoch nicht gelöst, da offenbar eine Art Sogkraft für eine weitere Nachfrage nach Parzellen entstand. So ist z.B. in einem anderen Dokument von Mai 1960 die Rede von mehr als 1000 neuen Anträgen und fehlendem Land und der daraus folgenden Notwendigkeit, die tatsächliche Bedürftigkeit der Antragsteller zu prüfen. ${ }^{181}$

Die hohen Zahlen an zugeteilten Parzellen in Samarkand stimmen zeitlich überein mit dem unionsweiten Höhepunkt des Bauprogramms - so waren 39,9 Prozent des gesamten Wohnraumbestandes im Jahr 1958 in individueller Hand. ${ }^{182}$ Wie bereits oben geschildert, erfuhr der individuelle Wohnungsbausektor in der zweiten Hälfte der 1950er-Jahre die größte Förderung und Verbreitung in der gesamten Sowjetunion, gefolgt allerdings von einer recht schnellen Umkehr ins Gegenteil. Die Entwicklung in Samarkand spiegelt in groben Zügen die unionsweite Politik wieder, jedoch mit eigenen Besonderheiten.

Im Rahmen des unionsweiten Wohnungsbauprogramms wurde der individuelle Wohnungsbau „unter Einbeziehung von Eigenmitteln der Bürger und unterstützt durch staatliche Kreditvergabe“ zu einem wichtigen Bestandteil der Beseitigung des Wohnraummangels erklärt und erfuhr auch ideologische Unterstützung. Ein Beitrag in einem „Agitatoren-Notizbuch“ fasst in routinierter Begeisterung Zahlen und Argumente zusammen: Demnach plante die UzSSR für die

178 SamOGA, f. 1617, op. 1, d. 62, 1. 67.

179 SamOGA, f. 1617, op. 1, d. 111, 1. 1-2.

180 SamOGA, f. 26, op. 1, d. 2168, 1l. 313, 322, 404.

181 SamOGA, f. 26, op. 1, d. 2355, 1. 476.

182 Smith 2010, S. 161. 
Jahre 1956-1960 fast genau so viel individuell gebaute Wohnfläche (nämlich 3 Millionen Quadratmeter) wie solche von staatlicher Hand (3,9 Millionen Quadratmeter); allerdings schließen diese Zahlen auch Wohnungsbauvorhaben auf dem Land mit ein. Es wurden die Vorzüge des neuen Systems gelobt: Eine große Rolle bei der Organisation, Verteilung und den bürokratischen Formalitäten hatten nun die Betriebe und andere Arbeitsstellen zu übernehmen, was das Prozedere erleichtern sollte. ${ }^{183}$ Des Weiteren wird die Übersetzung der oben bereits erwähnten Gorky-Methode in die traditionelle lokale Form der Nachbarschaftshilfe hashar gelobt und mit Beispielen von erfolgreicher Anwendung illustriert.

Diese positive Stimmung hielt allerdings nicht lange an, und zwar sowohl auf der lokalen als auch auf der Unionsebene. In Samarkand stieß die großzügige Verteilung von Land für individuelle Häuser zusammen mit anderen Bauprojekten offenbar schnell an ihre Grenzen. Bereits im Jahre 1958 wurde deutlich, dass das verfügbare Bauland angesichts des Bevölkerungswachstums nicht für den eingeplanten Umfang an staatlichem und individuellem Wohnungsbau ausreichte und dass weitere Flächen benötigt wurden. ${ }^{184}$ Im November 1960 fand eine Sitzung beim stellvertretenden Vorsitzenden des Gebiets-Ispolkom statt, bei der zahlreiche Verantwortliche der städtischen Partei-, Planungs-, Bau- und Verwaltungsorganisationen anwesend waren. Aus dem Sitzungsprotokoll geht hervor, dass im Jahre 1960 offenbar ein zwischenzeitliches Verbot der Erweiterung der Stadt auf Kosten von Kolchosenland verhängt worden war und dass in diesem Zusammenhang auch der individuelle Wohnungsbau gestoppt wurde. Damit wird auch die Notwendigkeit einer Revision bzw. Aktualisierung des neuen Generalplans begründet. ${ }^{185}$ Die Anordnung eines Verteilungsstopps wirkt verwirrend, wo doch noch wenige Monate zuvor, im Februar, der Gebiets-Ispolkom in einem seiner Beschlüsse die mangelnde Plansollerfüllung bei der Kreditvergabe für individuelle Häuser (lediglich 48 Prozent) und bei der Inbetriebnahme (77 Prozent) bemängelte. ${ }^{186}$ Offenbar galt das Zuteilungsverbot jedoch nur für kurze Zeit bzw. war mit genügend Ausnahmen versehen, denn bereits aus dem Jahr 1961 finden sich wieder zahlreiche Beschlüsse und Entscheidungen, die die Parzellenzuteilung bzw. den individuellen Hausbau betreffen.

Der Stimmungswechsel hing gewiss auch mit den oben erwähnten Gesetzesverschärfungen in Bezug auf den individuellen Wohnungsbau in größeren Städ-

183 Razykov, A. 1958. S. 11-18.

184 SamOGA, f. 1617, op. 1, d. 63, 1. 19-21.

185 SamOGA, f. 1617, op. 1, d. 63, 1. 36-38.

186 SamOGA, f. 1617, op. 1, d. 111, 1.1-4. 
ten zusammen. Nach dem unionsweiten Gesetz „Über individuellen und kooperativen Wohnungsbau" beschloss der Ministerrat der UzSSR im August 1962 ebenfalls den Übergang von individuellen Häusern mit nur einer Wohnung zum Bau von Etagenwohnhäusern unter Einbeziehung der Mittel der Bevölkerung in Form von Kooperativen. ${ }^{187}$ In einem Merkblatt von November 1962 werden Städte aufgezählt, darunter auch Samarkand, wo die Zuteilung von Parzellen zur individuellen Bebauung nur in Ausnahmefällen gestattet wurde, und zwar im Falle eines Abrisses wegen staatlicher Bauvorhaben. ${ }^{188}$ Offenkundig schlug sich in dieser Form eine Regelung nieder, die seit 1962 den Ministerräten der Republiken das Recht überließ, über die Vergabe von Parzellen und Krediten in bestimmten Städten selbst zu entscheiden. In den Hauptstädten sollte die Vergabe nach dem Gesetz komplett eingestellt werden.

Den Dokumenten ist ein deutlicher Rückgang der Parzellenzuteilung um die Wende des Jahrzehnts und zu Beginn der 1960er-Jahre zu entnehmen, und das, was dennoch verteilt wurde, ging - der Ausnahme entsprechend - vor allem an solche Personen, deren bisherige Häuser abgerissen werden mussten. ${ }^{189}$ Dabei wird die widersprüchliche Situation deutlich, in der sich städtische Verwaltung und Baubehörden befanden: Für die Errichtung von vier- bis fünfstöckigen Neubauten, diversen öffentlichen Gebäuden oder Straßen mussten Flächen, häufig im Stadtzentrum oder seiner Nähe oder auch in der Altstadt, frei gemacht werden, die in der Regel mit individuellen Häusern - alten oder neueren - bebaut waren. ${ }^{190}$ Die Besitzer bekamen neue Grundstücke in dafür vorgesehenen Vierteln der individuellen Bebauung am damaligen Stadtrand; paradoxerweise waren die neuen Grundstücke manchmal nur einige hundert Meter von den alten entfernt. ${ }^{191}$ Damit verlor die Stadt wertvolle Bauflächen, die an anderen Stellen sofort wieder bebaut wurden. Die Wohnungsnot verschärfte sich sogar kurzfristig, solange die

187 SamOGA, f. 1617, op. 1, d. 137, 1. 35. Diese Anordnung wiederholte im Wortlaut den Gesetzestext der Unionsebene.

188 SamOGA, f.1617, op.1, d. 150, 1. 24. Weitere Städte in der Liste sind Bukhara, Andidschan, Namangan - alle mit Bevölkerungszahlen um die 100.000.

189 SamOGA, f. 1617, op. 1, d. 161, 1. 18.

190 Ein großes Problem der Stadt und ein Teil des Konflikts zwischen Stadtverwaltung und Leitungen der Kolchosen bestand darin, dass die Kolchosen die Territorien in den so genannten „Projektgrenzen der Stadt“, die eigentlich frei bleiben sollten, bereits selbst zur Bebauung freigegeben hatten. Über die genauen Motive für diese Handlungen kann angesichts der beschränkten Quellenlage nur spekuliert werden. Eine mögliche Erklärung könnte sein, dass die Kolchoseleitungen sich davon eine Art „Schutzwall“ vor der Stadterweiterung versprochen hatten. Individuelle Bereicherung von konkreten Personen, die für die Zuteilung zuständig waren, ist ebenso wahrscheinlich.

191 Dies betraf z.B. Menschen in Viertel 1. 
neuen Wohnblöcke nicht fertig waren bzw. wenn es sich beim Neubau nicht um Wohnungsbau handelte. Einer meiner Interviewpartner hat es wie folgt zusammengefasst: „Samarkand wurde nur durch Abriss gebaut.“192 Gleichzeitig waren die entsprechenden Entscheidungen nicht immer koordiniert. Dazu trug auch die schwache Stellung des städtischen Ispolkom gegenüber den Interessen zum Beispiel der Industrie bei, die direkt den jeweiligen Ministerien unterordnet waren. John DiMaio konstatiert:

Most troubles of Soviet urban planning are a result of the sometimes fierce conflicts between the much overburdened but weak local Soviets and the nearsighted but powerful ministries and departments and their enterprises. (DiMaio 1974, S. 66)

Yulia Kosenkova spricht dazu von Exterritorialität der industriellen Betriebe. ${ }^{193}$ Die Kombination aus diversen Interessenskonflikten, fehlender Abstimmung bei der Planung und überstürzten Lösungen führte letzten Endes zu Verstößen gegen den Generalplan.

Im Sommer 1965 kam vom Ministerrat der UzSSR ein Beschluss, in dem die „nachlassende Aufmerksamkeit“ gegenüber dem individuellen Bausektor und damit sein Rückgang bemängelt und die Praxis des „unbegründeten“ Abrisses von Neubauten kritisiert wurde. Der UzGosstroi ordnete daraufhin an, binnen kürzester Zeit Pläne und Projektvorschläge für einen neuen individuellen Wohnungsbau zu erstellen. ${ }^{194}$ Der zwischenzeitliche Anstieg der Zuteilungen in Samarkand in den Jahren 1966-67 - in den beiden Jahren wurden mehr als 1500 Parzellen bebaut ${ }^{195}$ - ist mit Sicherheit darauf zurückzuführen. Gleichzeitig fanden in Fachzeitschriften und auf einigen Sitzungen Diskussionen über die Notwendigkeit einer Anpassung der sog. Typenprojekte (tipovoi proekt) ${ }^{196}$ sowohl an die lokalen klimatischen Bedingungen als auch an die traditionellen Bauweisen und die großen Familien statt. ${ }^{197}$ Die erneute Förderung des individuellen Wohnungsbaus kann auch mit dem Machtwechsel in der obersten politischen Etage

192 Interview mit einem ehemaligen bauverantwortlichen Parteimitarbeiter.

193 Kosenkova, 'Gradostroitel'noe myshlenie'.

194 SamOGA, f. 1617, op. 1, d. 195, 1. 88-89.

195 SamOGA, f. 1646, op. 2, d. 76, 11.1 und 4.

196 Ein tipovoi proekt war in der Regel ein standardisiertes Projekt eines Gebäudes (Wohnhaus, Schule, Kino, etc.). Nach dem Entwurf und dem Genehmigungsprozess wurden diese in speziellen Katalogen gesammelt und zur weiteren Anwendung an Projektinstitutionen verteilt. Sie dienten zur Kosteneinsparung und konnten mit oder ohne Anpassungen beliebig oft wiederverwendet werden.

197 Chebotareva 1974. Für einen Überblick über die Diskussion zur Einbeziehung von lokalen Baumethoden in die Projekte für industriellen Wohnungsbau siehe Kosenkova 2009. 
zusammenhängen - nach der Absetzung von Khrushchev 1964 vom Amt des Ersten Sekretärs der KPdSU wurde Brezhnev, dem auch eine wohlwollende Haltung gegenüber dem individuellen Wohnungsbau nachgesagt wird, zum Amtsinhaber auf Jahre hinaus. ${ }^{198}$

Der Zickzack-Kurs beschäftige offenbar einige Fachleute. In den Dokumenten der Architektur- und Planungsbehörde der Stadt für das Jahr 1966 findet sich eine selten kritisch ausformulierte Schrift vom Leiter der Behörde, A. Kogan, über die Perspektiven des Wohnungsbaus in Samarkand. ${ }^{199}$ Darin kritisiert er die langjährige Praxis der schlecht koordinierten individuellen Bebauung, in deren Zuge zufällige Gebiete in der Stadt für private Häuser vergeben wurden, weswegen neuer Massenwohnungsbau nur außerhalb der damaligen Stadtgrenzen und nur auf Kosten der übereigneten Kolchos-Ländereien möglich sei. Individuelle Bebauung in einer Großstadt wird von ihm als moral'no i fizicheski, also „im Geiste sowie physisch“, veraltet bezeichnet und zudem als sehr kostspielig gebrandmarkt, da sie im Vergleich zu einer dichteren Siedlungsform sehr hohe Kosten bei der Bereitstellung der Infrastruktur verursache, und zwar sowohl bei den Versorgungsleitungen für Wasser, Strom, Gas und Abwasser als auch bei der Ausstattung der Gebiete mit öffentlichen Einrichtungen und Verkehrsmitteln, so Kogan. Er plädiert für einen Stopp des individuellen Wohnungsbaus in Samarkand und für die Begrenzung des Flächenwachstums der Stadt. Er beklagt den „Hang der lokalen Bevölkerung zum [Leben am] Boden“ - gemeint damit ist die Bevorzugung eines eigenen Hauses mit Garten gegenüber einer Etagenwohnung durch viele Bewohner von Samarkand. Dies sieht Kogan als eines der größten Hindernisse auf dem Weg zum dringend anstehenden Umbau und zur Umgestaltung (rekonstruktsiya) der zentrumsnahen Stadtviertel.

Bezeichnend für die Widersprüchlichkeit der städtebaulichen Politik in Samarkand ist, dass in derselben (!) Akte ein Bericht zum erfolgten und geplanten Wohnungsbau in Samarkand enthalten ist, unterschrieben von Vertretern von Partei und Verwaltung, darunter auch Herrn Kogan. Demnach plante die Stadt für die Fünfjahresplan-Periode 1966-70, insgesamt 333.000 Quadratmeter an Wohnraum zu bauen, wobei fast ein Drittel (100.000 Quadratmeter) durch individuellen Wohnungsbau abgedeckt werden sollte. ${ }^{200}$ Beide Dokumente sind leider undatiert und haben auch keinen Empfänger im Briefkopf, sodass sich weder die Chronologie ihres Erscheinens nachvollziehen lässt noch klar wird, ob und in welcher Form diese miteinander in Konflikt stehenden Dokumente eine Wirkung

198 Rywkin 1980, S. 41-42.

199 SamOGA f. 1658, op. 2, d. 146, 1. 6-8.

200 SamOGA, f. 1658, op. 2, d. 146, 1. 1-5. 
entfalteten. Deutlich machen sie, dass der Wohnungsbau in Samarkand hinsichtlich der Folgen des individuellen Wohnungsbaus durchaus Diskussion und Widerspruch aus der städtebaulichen Perspektive provozierte. Darüber hinaus zeugen die Unterlagen von der Schwäche, die Einzelstimmen gegenüber Plänen hatten, die von zentralen Behörden angeordnet waren.

Parallel dazu wurde 1966 die Praxis der Übereignung von fruchtbaren Böden von den Kolchosen an die Städte zum Zwecke des Industrie- und Wohnungsbaus auf höchster Ebene massiv kritisiert und durch einen Erlass des Zentralkomitees der KPdSU und des Ministerrats der UdSSR strengen Auflagen untergeordnet. ${ }^{201}$ Damit wurde es für die Stadt in der zweiten Hälfte der 1960er- und am Anfang der 70er-Jahre deutlich schwieriger, Bauland zu bekommen. Zahlreiche Anträge auf Erteilung von Parzellen werden nun mit der Begründung abgewiesen, das Bauland reiche selbst für die vom Abriss Betroffenen nicht aus. ${ }^{202}$ Auch meine Gesprächspartner äußerten fast einstimmig die Beobachtung, dass es in den 70erJahren bereits sehr schwierig, ja beinahe unmöglich geworden war, eine Parzelle zu bekommen. Es ist sehr wahrscheinlich, dass diese Entscheidung unmittelbar mit dem Landkonflikt zwischen der Stadt und den umliegenden Kolchosen verbunden war.

Der Mangel an Bauland und widersprüchliche politische Entscheidungen schränkten den Handlungsspielraum der Stadtverwaltung und der Baubehörden von Samarkand immer weiter ein. Die Bautätigkeit der Stadt glich einem Schieberätsel-Spiel, bei dem die wenigen frei werdenden Flächen schnell bebaut wurden, um dem Bedarf auf irgend eine Weise nachzukommen, währenddessen für kurzfristige Lösungen die Vorgaben des Masterplans aufgegeben wurden. $\mathrm{Zu}$ diesem Umstand passt auch die Aussage eines Architekten, dass die Eigenheimviertel ursprünglich als temporäre Lösung gedacht waren und nur gebaut wurden, um die größte Not zu lindern, in Zukunft aber wieder abgerissen werden sollten, sobald genügend Geschoss-Wohnhäuser gebaut waren. ${ }^{203}$ Von diesem Interviewpartner wurde die Bemerkung allerdings mit dem Spruch „Es gibt nichts Beständigeres als etwas Temporäres“ eingeleitet. Im krassen Kontrast dazu war es nicht möglich, den von oben auferlegten Planvorgaben zu widersprechen: Ein anderer Zeitzeuge brachte die Widersprüche des Systems mit dem Zitat einer Losung auf den Punkt: „Der Plan ist Gesetz, die Erfüllung Pflicht, die Übererfüllung

201 SamOGA, f. 1617, op. 1, d. 210, 1. 88-90.

202 SamOGA, f. 1658, op. 1, d. 376, 1. 21.

203 Gespräch mit R.B. 
Ehre und die Qualität das Gewissen. “204 Dabei lassen sich die tatsächlichen Aushandlungsprozesse, formelle wie informelle, auf der Grundlage der vorhandenen Daten nur grob nachvollziehen. Zwar hatte ich die Chance, mit einigen Zeitzeugen zu sprechen, die Ende der 1960er und Anfang der 70er-Jahre Positionen in den städtischen Baubehörden innehatten, doch waren diese nicht gewillt, über entsprechende Details Auskunft zu geben, und bemühten sich vielmehr, die Frage des individuellen sowie des eigenmächtigen Wohnungsbaus in ihrer Bedeutung herunterzuspielen. ${ }^{205}$ Eine Ursache für die ausweichenden Antworten könnte die unmittelbare Verwicklung meiner Interviewpartner in Konflikte und Unregelmäßigkeiten im Zusammenhang mit eigenmächtigem Wohnungsbau in Samarkand sein, wie ein anderer Gesprächspartner mutmaßte - eine Einschätzung, die ohne konkrete Belege aber reine Spekulation bleibt.

Abschließend wurde im Jahr 1972 die Zuteilung von Parzellen für individuelle Bebauung eingestellt. Die einzigen Ausnahmen wurden Bürgern gewährt, deren Häuser zum Abriss bestimmt waren, sowie Invaliden des Zweiten Weltkriegs in schwieriger Wohnsituation. Bei neuen Bauvorhaben sollte darauf geachtet werden, dass möglichst wenig Wohnsubstanz dafür abgerissen wird. Die Leitung des städtischen Architektur-Planungsbüros wird unter der Androhung, persönlich zur Verantwortung gezogen zu werden, vor Verstößen gewarnt. ${ }^{206}$ Tatsächlich wurde der individuelle Wohnungsbau in den 1970er- und 80er-Jahren reduziert. Eine Rolle spielte dabei sicherlich, dass die Kapazitäten des industriellen Wohnungsbaus in dieser Zeit anwuchsen: Der Bau der Mikroraions A und B im Westen der Stadt sowie die erstmalige Errichtung von neunstöckigen Häusern konnten die Wohnungsnot schrittweise reduzieren.

\section{Der Weg zum Eigenheim}

Mark Smith beschreibt das Wohnungsbauprogramm als Teil des sowjetischen Wohlfahrtssystems, ${ }^{207}$ jedoch erforderte der individuelle Hausbau besonders viel private Initiative und die persönliche Bereitschaft, beachtliche Finanzen und Arbeit $\mathrm{zu}$ investieren. Zudem existierte in Samarkand eine zahlenstarke Kategorie von Menschen, die das Grundstück vom Staat nicht nach einem Antrag, sondern

204 Gespräch mit O.P.

205 Aufgrund des Datenschutzes kann ich mir hier nicht erlauben, die Position der Person zu nennen, da sonst Rückschlüsse auf die Identität leicht möglich sind. Strikte Anonymität war aber von den Interviewpartnern explizit gewünscht.

206 SamOGA, f. 1658, op. 1, d. 355, 1. 57.

207 Smith 2010, S. $139 \mathrm{ff}$. 
als Ersatz bekamen - nachdem nämlich das eigene Haus staatlichen Baumaßnahmen zum Opfer gefallen war und sie ihr Haus von Null wieder aufbauen mussten. Hausbau war also nicht immer eine freiwillige Entscheidung; er konfrontierte Menschen in womöglich sehr unterschiedlichen Lebenssituationen mit einer herausfordernden Aufgabe. Zu den bürokratischen Hürden bei der Beantragung kamen diverse Auflagen und Restriktionen, Kosten und Knappheit von Baumaterial sowie Engpässe in der infrastrukturellen Versorgung. Je nach finanzieller und sozialer Positionierung entwickelten die Bewohner von Samarkand unterschiedliche Strategien, um diese Herausforderungen zu meistern. Um die persönliche Perspektive einzufangen, habe ich neben dem Studium des Archivmaterials, aus dem primär die bürokratischen Vorgänge ersichtlich sind, Interviews mit den Bewohnern des „privaten Sektors“ geführt, aber auch mit Menschen, deren Familie kein Haus bauen konnte oder wollte. In den folgenden Abschnitten werde ich versuchen, beide Perspektiven darzustellen; dabei wird der Fokus zuerst auf der regulären Form liegen, auf dem „planmäßigen individuellen Wohnungsbau“ (planovoe individual'noe stroitel'stvo). Der parallel dazu existierende semi-legale oder gar illegale sogenannte „eigenmächtige“ (samovol'noe) Hausbau wird in seinen unterschiedlichen Formen und Ausprägungen in Kapitel 5 ausführlich besprochen.

Die meisten Gespräche, nämlich vier längere Interviews und fünf kürzere Gespräche (letztere mit Menschen, die vor ihren Häusern saßen), habe ich mit Bewohnern des Viertels 1 (Abb. 1) geführt, das ich aufgrund seiner offensichtlich planmäßig angelegten Straßenführung als Beispiel ausgesucht hatte. ${ }^{208}$ Weitere drei Gespräche wurden im Viertel 2 geführt. Für fast alle Gesprächspartner gilt, dass die Häuser bereits von ihren Eltern gebaut wurden, sodass ihnen als Kindergeneration das Wissen bzw. die Erinnerung zu vielen Details fehlte, was die Daten stark fragmentarisch macht.

208 Im Gegensatz zu den meisten Vierteln mit individueller Bebauung, die im einfachen Raster angelegt sind, macht der Straßenverlauf hier den Eindruck, dass intensivere Planungsüberlegungen am Werk waren. Die untersuchten Viertel haben keine offiziellen Namen, und werden hier neutral als Viertel 1 und 2 bezeichnet. 


\section{Das bürokratische Prozedere}

Die formelle Seite der Beantragung einer Parzelle unterschied sich in Samarkand im Wesentlichen nicht von dem, was für die gesamte UdSSR galt. ${ }^{209}$ Parzellenzuteilung erfolgte über eine Warteliste bei der Arbeitsstelle oder über die spezielle Abteilung beim Stadt-Ispolkom.

Um auf diese Liste zu gelangen, musste man einen Antrag stellen und eine Fürsprache von Seiten des Arbeitgebers, einen Nachweis über das Beschäftigungsverhältnis sowie eine Bescheinigung des Hauskomitees (Domkom) über die Größe der Familie und des vorhandenen Wohnraumes beibringen. ${ }^{210}$ Ein Platz auf der Liste garantierte jedoch nicht immer ein Grundstück, denn, wie bereits deutlich geworden sein sollte, variierte die Landverfügbarkeit je nach Jahr stark; zudem blieb die Zahl der Bauwilligen stets höher als die möglichen Kapazitäten erlaubten, sodass die Wartezeit mehrere Jahre betragen konnte. So wurden zum Beispiel zahlreiche Anträge aus dem Jahr 1953 erst im Jahr 1957 genehmigt. ${ }^{211}$ Das Fehlen von einheitlichen Listen beim Stadt-Ispolkom, ein verbreitetes Problem, das sowohl in Samarkand als auch in anderen Städten der Sowjetunion beklagt wurde, bot viel Raum für Fehler und Manipulationen. Das Zurückgreifen auf informelle Praktiken, um auf der Warteliste vorzurücken oder anderweitig begünstigt zu werden, lässt sich auf der Grundlage der von mir gesammelten Daten nur für vereinzelte Beispiele sicher feststellen, ansonsten nur vermuten. ${ }^{212}$ Gleichzeitig kann ein größeres Ausmaß der Nutzung von informellen Praktiken wie blat, Vetternwirtschaft oder Bestechung nicht ausgeschlossen werden, eine dahingehende Behauptung könnte sich jedoch nur auf die große allgemeine Verbreitung dieser Phänomene in der SU stützen. ${ }^{213}$ Eine Fokussierung auf solche Praktiken ist nicht Ziel dieser Arbeit, vielmehr betrachte ich sie als selbstverständlichen Teil des täglichen Lebens in der Sowjetunion, der sowohl die Konsumbedürfnisse befriedigen half als auch das Funktionieren des Systems ermöglichte.

Eine legale Alternative zur Warteliste war für Wohlhabende bzw. für Menschen mit Ersparnissen der Kauf eines Hauses. Wie bereits erwähnt, durfte das Grundstück selbst dabei nicht offiziell zum Gegenstand des Handels werden, und

209 Auch die Sprache der Bürokratie und der bürokratischen Vorgänge, wie z.B. Antragstellung, war Russisch. Von den bei der Archivrecherche gefundenen Dokumenten war nur ein verschwindend geringer Teil nicht auf Russisch verfasst, sondern auf Usbekisch oder Tadschikisch.

210 SamOGA, f. 1617, op. 1, d. 65, 1. 102.

211 SamOGA, f. 26, op. 1, d. 1972, 1. 25.

212 SamOGA, f. 26. op. 1, d. 1978, 1. 2-5: Der Fall beschreibt eine Familie, die sich auf unterschiedlichen Wegen ganze sechs benachbarte Häuser bauen konnte.

213 Siehe dazu z.B. Ledeneva 1998 oder Ledeneva (Hg.) 2018, S. 40-46. 
daher wurde in so einem Fall ein angefangenes Haus oder zumindest ein Fundament, das schon errichtet war, gekauft bzw. das Kaufobjekt derart maskiert. ${ }^{214}$ Solche verschleierten Käufe waren den Archivquellen wie auch mündlichen Erinnerungen zufolge offenbar verbreitet und den Behörden bekannt. Diese hatten aber offensichtlich wenig Mittel, die Vorgänge intensiver zu kontrollieren oder zu unterbinden. Ein Interviewpartner, dessen Familie selbst kein Haus hatte, erzählte von der weit verbreiteten Praxis des Kaufens, wobei die in den Dokumenten festgehaltene Summe immer viel niedriger war als die tatsächlich gezahlte, weil es auch für Kaufpreise festgelegte Obergrenzen gab. ${ }^{215}$

Die Größe der Grundstücke variierte: Für Anfang der 1950er-Jahre sind großzügige $600 \mathrm{~m}^{2}$ charakteristisch, die später, offenbar weil Land immer knapper wurde, kleiner werden und sich auf 500 bis $300 \mathrm{~m}^{2}$ reduzieren. Aus diesem Grund hatten Parzellen an den zwei Seiten einer Straße häufig unterschiedliche Größe.

Mit einem positiven Zuteilungsbescheid sollte sich der Antragsteller bei der städtischen Architektur- und Planungsbehörde melden, um dort ein vorhandenes individuelles Projekt genehmigen zu lassen oder, was eher die Regel war, ein Typenprojekt zugewiesen zu bekommen. Das Typenprojekt bestand aus einem Haus mit quadratischem (ca. 10 x 10 Meter) oder rechteckigem (ca. 10 x 8 Meter) Grundriss und umfasste 2-4 Zimmer (Abb. 6). Dieser Typ bzw. seine Variationen erfreuten sich (offensichtlich mangels Alternative) großer Beliebtheit. ${ }^{216}$ Die Projekte wurden, wie es hieß, an die „lokalen Baumaterialien und lokalen klimatischen Bedingungen“ angepasst; dies bezog sich vor allem auf die Baumaterialien, nicht aber auf die Bauform (traditionelle Hofhäuser). ${ }^{217}$ Errichtet werden sollten die Mauern, mit Ausnahme des Fundaments, aus ungebrannten Lehmziegeln, und das Gebälk sollte aus lokalen Holzarten hergestellt werden.

214 Dies war bei zwei der Befragten der Fall. Auf die Frage, wie denn der Kauf genau funktionierte, konnten sie keine präzise Antwort geben (mit Verweis auf ihr junges Alter zu dem Zeitpunkt) und stellten dann Vermutungen an. Für die Richtigkeit ihrer Annahmen spricht, dass in beiden Fällen eine „halbe“ Parzelle bebaut war - jeweils $300 \mathrm{~m}^{2}$ (Gespräch mit A.N. sowie A.O). 215 Gespräch mit A.S.

216 Babakhanov 1960, S. 33 gibt an, dass dieses Typenprojekt bereits vor dem Zweiten Weltkrieg entworfen worden war und in großer Zahl Verwendung fand. Obwohl bei individuellen Häusern fünf Zimmer erlaubt waren, betrug die Zimmerzahl bei den Typenprojekten maximal vier.

217 Betrachtet man heute die Eigenheimviertel im Satellitenbild oder auch bei Begehung, so fällt auf, dass aus den ursprünglichen „Würfelhäusern“ zum großen Teil Hofhäuser geworden sind. Für eine Beschreibung der Typologie und des Wandels solcher Eigenheim-Quartiere in Taschkent siehe Gangler et al. 2006, S. 154-167. 


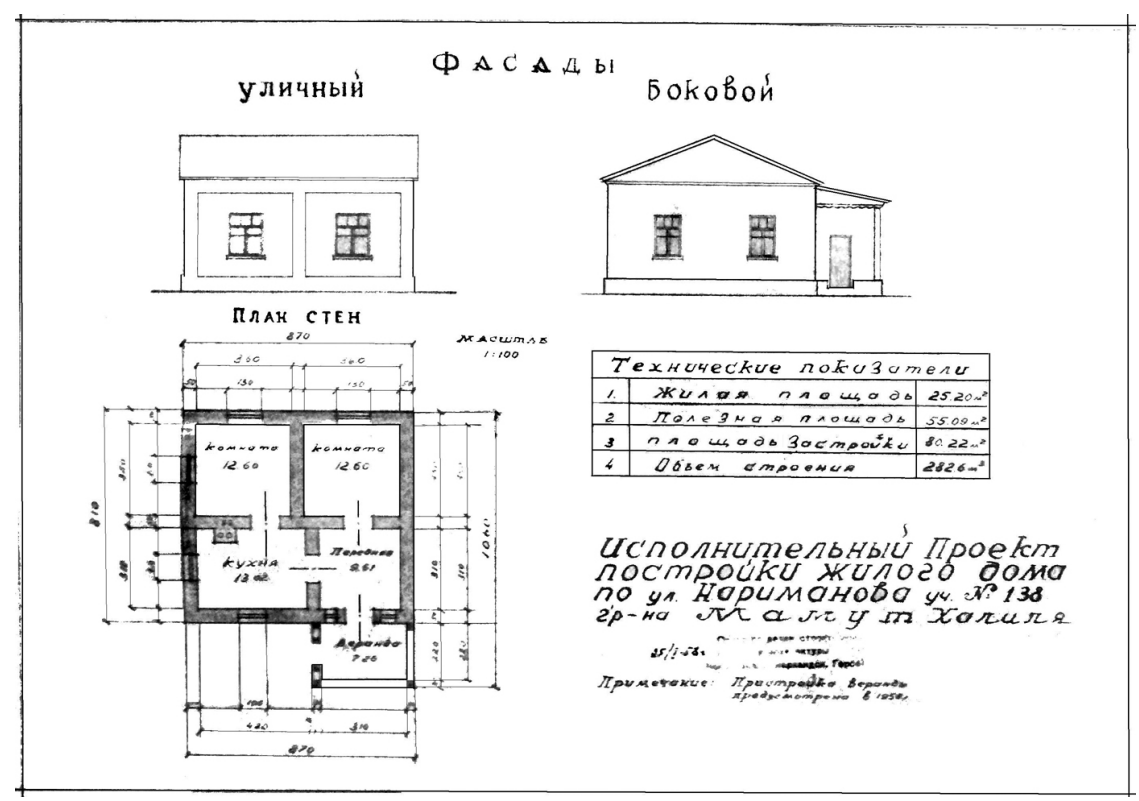

Abb. 6: Typenprojekt für ein individuelles Haus in Samarkand. Foto: Autorin, bearbeitet.

Der ökonomische Faktor dürfte bei der Wahl konventioneller Baumaterialien eine große Rolle gespielt haben, da Lehmziegel ohne industriellen Einsatz selbst hergestellt werden konnten. In Zuschnitt und Anordnung der Räume unterschieden sich die Typenprojekte nicht wesentlich von denen, die z.B. für die Belarussische SSR entworfen wurden (Abb. 7).

Die Rücksichtslosigkeit gegenüber lokalen Bautraditionen und Familienstrukturen bei der Ausarbeitung von Typenprojekten für die Städte Usbekistans war recht charakteristisch für die 1950er- und frühen 60er-Jahre. Man kann sie deuten als eine Mischung aus einerseits Ignoranz seitens der Planer und andererseits einer bewussten ,zivilisatorischen“ Herangehensweise, die bestimmte Arten des Wohnens als progressiv und andere als rückständig einstufte. So wurde z.B. das Vorhandensein von Fenstern und Holzfußböden und die Verwendung von neuartigen Baumaterialien in traditionellen Wohnhäusern durch sowjetische Ethnografen als Zeichen für die erfolgte „Anhebung des kulturellen Niveaus“ gewertet. ${ }^{218}$

218 Zhilina 1978, S. 97-98. 


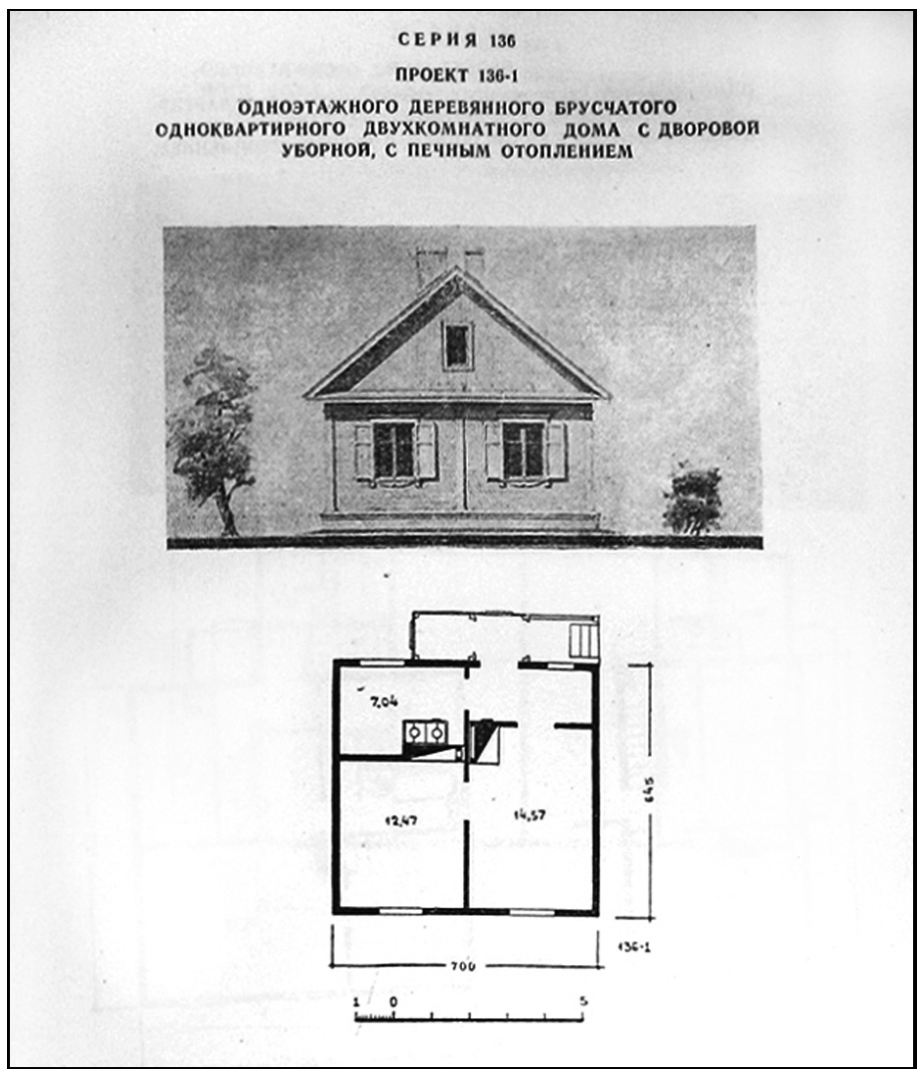

Abb. 7: Typenprojekt für ein individuelles Haus, Belarussische SSR.

Quelle: publiziert auf https://su-maloetazhki.livejournal.com/150850.html

\section{Finanzierungsmöglichkeiten}

Wenn eine Finanzierungshilfe gebraucht wurde, konnte ein Antrag auf Kreditzuteilung gestellt werden. Die Stadtbewohner konnten je nach Berufsgruppe 50\% bis 70\% (letzteres für Ärzte) der Baukosten in Form eines Kredits finanziert bekommen. Gleichzeitig durfte die Höchstsumme nicht über 5.000 bzw. 7.000 Rubel (vor der Währungsreform von 1961) respektive 500 bzw. 700 Rubel (danach) 
betragen. ${ }^{219}$ Die höchstmögliche Baukostensumme war also mit 10.000 bzw. 1000 Rubel angesetzt. Inwiefern dieser Ansatz realistisch war, ist fraglich - ein internes Informationsblatt des Präsidiums der KPdSU aus dem Jahr 1957 schätzt, dass diese Summe lediglich 20-25\% der Kosten für ein „Ein-Wohnungs-Haus“ (odnokvartirnyi dom) ausmachte. ${ }^{220}$ Meine Gesprächspartner reagierten auf die Frage nach der Höhe des Kredits in der Regel mit Abwinken, begleitet von Bemerkungen wie der, dass es „selbstverständlich zu wenig“ war. Einer der Interviewpartner, der als Jugendlicher an einem Hausbau beteiligt war, berichtete, die Familie habe auf den Kredit verzichtet, um keine Schulden anzuhäufen. ${ }^{221}$ Die meisten anderen Gesprächspartner bestätigten, dass ihre Eltern Kredite aufgenommen hätten, zum Beispiel bei der Teefabrik, konnten sich aber an die Höhe und weitere Details nicht erinnern. Offiziellen Bestimmungen zufolge wurde für den Kredit ein Jahreszins von 2\% festgelegt; der Berufsgruppe entsprechend musste der Kredit innerhalb von sieben bis zehn Jahren quartalsweise in gleichen Raten zurückgezahlt werden. Bei zu später Rückzahlung wurde ein Strafzins von 3\% auf die nicht beglichene Summe erhoben. Beantragung und Vergabe von Krediten erfolgte entweder - wie oben im Fall der Teefabrik - über den Arbeitgeber oder direkt bei der Kommunalbank. ${ }^{222}$

Trotz der finanziellen Unterstützung ging mit einer Parzellenzuteilung nicht automatisch die Möglichkeit einher, tatsächlich ein Bauvorhaben umzusetzen. Gesundheitliche oder finanzielle Probleme konnten dazu führen, dass die Menschen die Zuteilung gar nicht nutzen konnten. Häufig wird in den Dokumenten thematisiert, dass das bürokratische Prozedere nicht bis zu Ende geführt wurde, was den Verlust des Rechts auf Bebauung nach sich ziehen konnte. ${ }^{223}$

\section{Zuteilung nach Abriss}

Eine eigene Kategorie der Parzellenempfänger waren die von Abriss Betroffenen. Die Gesamtzahl bzw. der Anteil dieser Fälle für den betrachteten Zeitraum lässt sich nicht genau ermitteln, doch die Häufigkeit, mit der das Thema und entspre-

219 Verordnung Nr. 320 des Finanzministeriums der UdSSR vom 16. Mai 1955 „Über die Festlegung von Regeln für die Kreditvergabe für individuellen Wohnungsbau seitens der Kommunalbanken und der Landwirtschaftsbank“, SamOGA, f. 26, op. 1, d. 1809, 1. 16-26.

220 Protokolle des Präsidiums der KPdSU, Bd. II, S. 746.

221 Gespräch mit H.K.

222 SamOGA, f. 26, op. 1, d. 1809, 11. 16-25.

223 SamOGA, f.26, op. 1, d. 1675, 1. 43. 
chende Zuteilungsbescheide in den Dokumenten des Stadt-Ispolkom auftauchen, erlaubt, ihre Zahl recht hoch anzusetzen. Unter den von mir befragten Menschen machen sie fast die Hälfte aus. Einige von den Geschichten möchte ich kurz schildern. Das Bemerkenswerte dabei ist, dass insbesondere im Viertel 1 die neuen Parzellen wenige hundert Meter Luftlinie von den alten Häusern entfernt zugeteilt wurden. Dies war z.B. der Fall bei Herrn Salovatov. Sein Vater hatte sich im Jahr 1951 von seinem als Bergbauarbeiter in Isfara verdienten Geld eine Haushälfte an der Karl-Marx-Straße gekauft. Doch bereits 1954 musste das Haus dem Ausbau der Straße weichen. Die Familie erhielt daraufhin eine Parzelle auf der Michurin-Straße. ${ }^{224}$ Drei weitere Beispiele deuten auf eine Umsiedlung von mehreren Familien, die in kleinen Gehöften um die heutige Gagarin-Straße wohnten. Als Toponym vor allem für die Gegend um die Kreuzung Gagarin-/Mirzo Ulughbek (damals Karl-Marx)-Straße ist die persischsprachige Bezeichnung Boghi-Shamol gebräuchlich. Drei von den Personen, die ich befragt habe, lebten dort mit ihren Familien und gehören der Volksgruppe der Eroni an. ${ }^{225}$ Sie hatten Häuser und Gärten, teilweise als Teil der Kolchose, bevor der Abriss erfolgte. Ihre neuen Häuser stehen nun weniger als ein Kilometer von den alten entfernt. ${ }^{226}$

Theoretisch konnten Menschen zur Kompensation nach Abriss statt eines Grundstücks eine der so genannten „sanitären Norm“ von $9 \mathrm{~m}^{2}$ pro Person entsprechende Wohnung bekommen. Doch in den 1950er-Jahren, bevor der Massenwohnungsbau einsetzte, war dies für Samarkand praktisch gar nicht möglich. Auch später, in den 1960ern, entschieden sich viele für ein Haus mit einen Stück Land, sei es weil die standardisierten 2- oder 3-Zimmer-Wohnungen zu klein waren für die großen Familien, oder auch wegen der Möglichkeit, auf dem Grundstück etwas anzubauen oder kleine Tiere wie Hühner, Schafe oder gar Schweine zu halten, ${ }^{227}$ was ebenfalls Bedeutung für die wirtschaftliche Lage der Familie hatte. Auf die Frage, wieso sie keine Wohnung wollten, antworteten fast alle, dass es „auf dem Boden“ (na zemle) besser sei. Und natürlich dürfen wir nicht vergessen, dass ein Haus mit begrüntem und schattigem Innenhof viel angenehmere mikroklimatische Eigenschaften für die langen heißen Sommermonate hatte als eine Wohnung in einem Plattenbau. In den traditionellen Hofhäusern wird der Innenhof als erweitertes Wohn- bzw. Aufenthaltsraum für die warme

224 Gespräch mit Herrn Salovatov (Klarname und Verzicht auf Tonaufnahme auf ausdrücklichen Wunsch des Interviewpartners).

225 In Samarkand gab es mehrere Eroni-Siedlungen; eine davon - Panjob - existiert noch heute, von der Stadt längst verschluckt.

226 Zur Orientierung siehe Abb. 8.

227 Die Frau von Herrn Salovatov berichtete, dass ihre Familie, die seit 1958 ebenfalls im Viertel 1 wohnte, ihr Einkommen durch eine kleine Schweinezucht aufbesserte. 
Jahreszeit benutzt - damit konnte auch der geringe überdachte Wohnraum ausgeglichen werden. ${ }^{228}$ Insbesondere für lokale, alt-einheimische tadschikisch- und usbekischsprachige Familien ${ }^{229}$ war das Vorhandensein bzw. die Möglichkeiten für den Anbau eines Empfangs- oder Gästezimmers wichtig, wo man Besuch empfangen konnte, ohne den Rest des Haushaltes zur Schau stellen zu müssen. ${ }^{230}$

Bei einem Abriss stand den Bewohnern neben einem Grundstück auch eine Kompensationszahlung im Wert ihres alten Hauses zu. Bestimmt wurde dieser Wert durch eine Kommission, die nach existierenden Tarifen abzüglich eines Verschleißkoeffizienten die Entschädigung festlegte. Die Zahlungen bezogen sich auf die offiziell festgelegten Preise für das Baumaterial, die allerdings offenbar weit unter den realen Preisen lagen, was die Betroffenen in eine schwierige Lage brachte. Eine solche Situation schildern im Jahr 1963 mehrere von Abriss betroffene Einwohner der Altstadt in einem Beschwerdebrief an Leonid Brezhnev, der damals Vorsitzender des Präsidiums des Obersten Sowjets der UdSSR und damit das formelle Oberhaupt des Staates war. ${ }^{231}$ Bezeichnend ist, dass die Bittsteller dabei nicht das Bauvorhaben und die Umgestaltung der Stadt hinterfragen oder kritisieren, sie loben es sogar als ein Zeichen des „politisch-kulturellen und wirtschaftlichen Wachstums“. Sie fordern aber eine vernünftige Entschädigung oder Erstattung, die ihnen ermöglichen würde, einen den „Familienbedingungen entsprechenden“ Wohnraum für sich selbst neu aufzubauen. Eine Reaktion darauf liegt der Akte in Form eines Telegramms bei. Darin wird angeordnet, die Möglichkeit zu prüfen, den Betroffenen Baumaterial zu staatslimitierten Prei$\operatorname{sen}^{232}$ zur Verfügung zu stellen. Ob die Beschwerde letztlich zu Gunsten der Verfasser des Briefs ausgegangen ist, lässt sich leider nicht nachvollziehen; der Vorgang illustriert jedenfalls die verbreitete Praxis der Beschwerdebriefe „nach oben“, d.h. an sehr hohe Instanzen. Dies war häufig das letzte Mittel und auch

228 Diese Beobachtungen haben auch Architekten von TashZNIIEP gemacht, und versucht in experimentellen Projekten umzusetzen (Kontorer 1971, S. 35); Michail Rywkin relativierte damit die im unionsweiten Vergleich geringe durchschnittliche Wohnfläche pro Person, da die offiziellen Zählungen nur den überdachten Wohnraum rechneten (Rywkin 1989, 40).

229 Damit meine ich diejenigen Familien oder deren Vorfahren, die vor der russischen Eroberung oder der Oktoberrevolution in Samarkand gelebt haben.

230 Dieses Bedürfnis, so scheint es, war vielen Architekten und vor allem denjenigen, die sich nicht mit lokalen Traditionen auskannten (weil sie entweder in einer russischsprachigen Umgebung aufgewachsen waren, oder aus anderen Teilen der Sowjetunion in die Republik gekommen waren) lange Zeit nicht bewusst. Der Architekt Pulatov versuchte seine Kollegen in dieser Hinsicht aufzuklären (Pulatov 1971, S. 17-19).

231 SamOGA, f. 26, op. 1, d. 2526, 1. 42-44.

232 D.h. zu Preisen für die staatlichen Organisationen und Betriebe, die niedriger waren als die in staatlichen Verkaufsstellen für Verbraucher angesetzten. 
die letzte Hoffnung, die als ungerecht empfundene Behandlung seitens der lokalen Behörden zu beenden und das eigene Anliegen durchzusetzen.

In diesem Beschwerdebrief wird noch ein anderes Problem angemahnt: die Jahreszeit, zu der die Häuser abgerissen und mit dem Bau der neuen angefangen werden sollte. Offenbar waren die Betroffenen mit der Situation konfrontiert, den Bau in den Herbstmonaten vollenden zu müssen, um für den Winter ein Dach über dem Kopf zu haben - ihren Schilderungen zufolge ein unrealistisches Vorhaben, da allein die Herstellung der Ziegel mindestens einen Monat dauert und diese im Herbst nicht gut austrocknen können. Daraus ein Haus zu bauen, würde ein Leben in feuchten Räumen bedeuten und bei den Bewohnern zu Krankheiten führen. Eine Reaktion darauf bleibt uns verschlossen, aber der Vorgang weist uns auf die Unsicherheit und die Mühen hin, die von Abriss Betroffene ertragen mussten.

Der bereits erwähnte Herr Salovatov berichtete davon, dass er mit seiner vierköpfigen Familie einen Winter lang ein Zimmer bei Nachbarn mieten musste, weil das Haus noch nicht fertig war. Nach der regulären Zuteilung aus der Warteliste standen die Menschen nämlich häufig vor der Situation, die von ihnen bisher bewohnten staatlichen Wohnungen schnell verlassen zu müssen, da der Staat den Wohnraum frei haben wollte. ${ }^{233} \mathrm{Da}$ aber der Bau in der Regel noch gar nicht begonnen hatte bzw. zumindest nicht vollendet war, mussten die Betroffenen ein provisorisches Dach über dem Kopf finden. Das stellte zusätzliche Strapazen dar, wie das Wohnen in temporären Hütten (vremyanki) oder die Anmietung eines Zimmers, oder es erforderte, einen bürokratischen Kampf zu führen, damit man vorerst noch in der staatlichen Wohnung bleiben konnte.

\section{Das Bauen}

Der Bauprozess gestaltete sich nach übereinstimmenden Erinnerungen sehr langsam. In der Regel versuchten die Menschen, möglichst schnell, vorzugsweise während der Sommermonate, den Rohbau mit einem Zimmer fertig zu bekommen, in dem die Familie wohnen konnte. Der Rest wurde je nach Möglichkeiten nach und nach, häufig über mehrere Jahre hinweg, fertiggestellt, aus- oder umgebaut. Der Vertrag, der bei einer Parzellenzuteilung abgeschlossen wurde, enthielt Auflagen bezüglich Fristen, zu denen bestimmte Bauvorgänge abgeschlossen werden sollten. Er nahm offenbar auch Rücksicht auf die langsame Geschwindig-

233 SamOGA, f. 26, op. 1, d. 1972, 1. 43. 
keit - insgesamt gewährte der Vertrag drei Jahre für die komplette Fertigstellung. ${ }^{234}$ Demnach musste im ersten Jahr das Fundament gelegt werden, im zweiten Jahr mussten mindestens 50 Prozent des Baus fertiggestellt werden, und zum Ende des dritten Jahres sollte der Bau abgeschlossen sein. Im Anschluss sollte er von einer Kommission abgenommen werden.

Neben den finanziellen Einschränkungen stellte die Knappheit bestimmter Baumaterialien, vor allem von Holz und gebrannten Ziegeln, die häufig für das Fundament verwendet wurden, ein großes Problem dar. So gab es zum Beispiel Einschränkungen in Bezug auf die Stückzahl (2.000) der Backsteine, die pro Jahr gekauft werden durften; auch für den Kauf von Holz musste eine spezielle Genehmigung besorgt werden. Der Arbeitgeber bestätigte den wirklichen Bedarf und es existierten Kontrollen, die die Einhaltung der Vorgaben und die Rechtmäßigkeit des Erwerbs überprüften. Einige der Informanten erinnerten sich, dass es bei ihren Eltern Kontrollen gab, bei denen die Quittungen für das gekaufte Baumaterial überprüft wurden, weshalb sie diese für den Fall einer Kontrolle noch jahrelang aufbewahrten. Angesichts des Mangels an Baumaterial sollten Bauteile aus den abgerissenen Häusern - vor allem Holzbalken - wiederverwendet werden, ${ }^{235}$ ihre Mitnahme war offiziell erlaubt. ${ }^{236}$ Bestimmte Kontingente an Baustoffen wurden über die Arbeitsstellen verkauft, vorausgesetzt deren Leitung bemühte sich, sie $\mathrm{zu}$ besorgen. In der Regel waren das Betriebe, die bereits für ihre Mitarbeiter Grundstücke bekommen hatten. Bestimmte Personenkategorien wie Kriegsveteranen, Militärs usw. erhielten Vergünstigungen.

Das hauptsächliche Baumaterial für die Hauswände - Lehm für ungebrannte Lehmziegel - war praktisch überall verfügbar. Den Lehm hoben die Menschen häufig gleich auf dem eigenen Grundstück aus, die Ziegel wurden unter Beihilfe von Nachbarn oder Verwandten selbst hergestellt oder es wurden dafür Arbeiter bezahlt. Nach Berichten kosteten 1000 ungebrannte Lehmziegel in den 1970erJahren nur 14 Rubel (ein günstiger Preis gegenüber 120 Rubel für dieselbe Menge von industriell hergestellten Backsteinen), was diese Art des Bauens erschwinglich machte.

Je nach Fähigkeiten und finanziellen Möglichkeiten und je nach dem, wie komplex bzw. aufwändig die Arbeiten waren, führten die Menschen diese selbst aus oder engagierten Baumeister - letzteres z.B. für den Bau von Dach oder Fundament. Andere bezahlten privat organisierte Baubrigaden. In den beschriebe-

234 SamOGA, f. 1658, op. 1, d. 376, 1. 29-30.

235 Babakhanov 1960, S. 25.

236 SamOGA, f. 1617, op. 1, d. 136, 1. 55-57. 
nen Jahren scheint Samarkand einer einzigen Baustelle geglichen zu haben, sodass leicht vorzustellen ist, dass Bauarbeiter oder einfach bautechnisch erfahrene Bürger sich in dieser Weise zusammenschlossen, um etwas dazuzuverdienen. Ebenso entwickelt war das System der Tagelöhner (mardikor). Dazu kam Hilfe von Freunden bzw. Verwandten oder „Landsleuten“, ${ }^{237}$ auch im Sinne der bereits angesprochenen Form der Nachbarschaftshilfe hashar.

In den Berichten des Stadt-Ispolkom findet man in regelmäßigen Abständen Kritik und Klagen über die unzureichende Kontrolle über den Bauvorgang. Offenbar hielten sich nicht alle Bauherren an die festgelegte Norm ( $9 \mathrm{~m}^{2}$ pro Person), sondern bauten größere Häuser, oder sie umgingen die Regeln auf andere Art. Den Erinnerungen der Privatpersonen zufolge schwankte die Strenge der Kontrollen tatsächlich sehr und bezog sich vor allem auf die zur Straße zeigende Hausfront. Auch heute noch erkennt man teilweise die Häuser aus dieser Zeit an der bestimmten Fenstergröße und an der Traufhöhe.

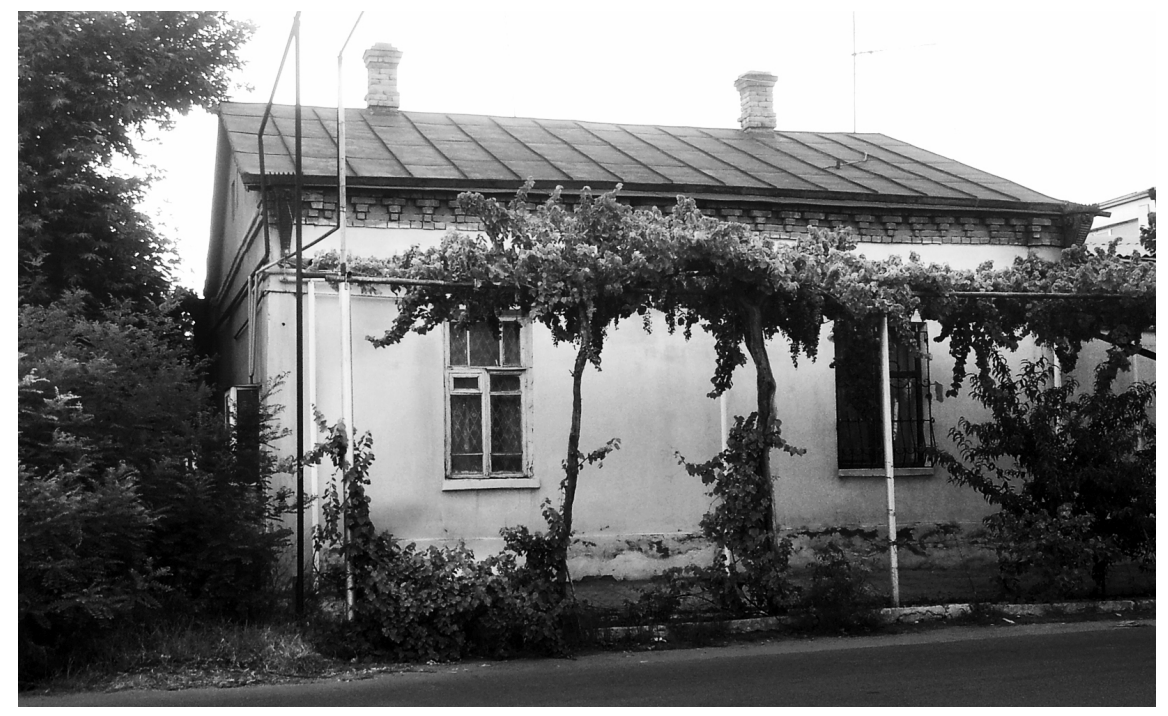

Abb. 8: Ein Typenhaus heute in Viertel 1. Foto: Autorin.

Im Inneren der Parzelle existierte offenbar viel mehr Freiheit, was Anbauten betraf, sodass viele die Wohnfläche der standardmäßig nicht sehr großen Typenhäuser erweiterten. In der Theorie bedurften solche Erweiterungen einer vor-

237 Dies galt z.B. für die Gruppe der Krimtataren, eine 1944 von der Krim in die sowjetischen Zentralasienrepubliken deportierte turksprachige Gruppe. 
herigen Genehmigung durch die Bauabteilung des Stadt-Ispolkom. Zahlreiche Anbaugenehmigungen zeigen aber, dass es kein großes Problem darstellte, solche rückwirkend zu erhalten, da die „Verbesserung der Wohnsituation“ für Familien mit beachtlicher Größe von 5-10 Mitgliedern stets als gewichtiger und legitimer Grund galt. ${ }^{238}$ All das zeugt von einer relativ großen Autonomie, die die Menschen genossen, wenn sie innerhalb des vorgegebenen Rahmens verblieben.

\section{Die Infrastruktur im privaten Sektor}

Da die Parzellen in den Vierteln in der Regel etappenweise vergeben wurden, standen die neuen Häuser häufig zuerst buchstäblich am Feldrand, der womöglich erst einige Jahre später in Bauland umgewandelt wurde. Ohne Ausnahme erinnerten sich alle Gesprächspartner an „Obstgärten, Weinreben und Felder“ bzw. an Gehöfte, die die neuen Häuser umgaben bzw. noch kurz davor an deren Stelle standen.

Auch bedeutete die Fertigstellung eines Hauses keine Garantie für eine umgebende Infrastruktur. Elektrischer Strom war nicht immer sofort verfügbar, blieb dann aber lange Zeit die einzige infrastrukturelle Versorgung überhaupt, die die Stadt zur Verfügung stellte. Wasser mussten sich die Bewohner die ersten Jahre an öffentlichen Wasserpumpen oder aus den Bewässerungsgräben besorgen. Gasleitungen wurden erst ab Mitte der 1960er-Jahre gelegt, während die Kanalisation erst Jahrzehnte später gebaut wurde, wenn überhaupt. So ließen die Einwohner der ehemaligen Michurin-Straße im Viertel 1 selbst durch eigene Investitionen Mitte der 1980er-Jahre einen Anschluss an die städtische Kanalisation bauen: „Dies von der Stadt zu erwarten war vergebens, da der private Sektor für die Stadt sowieso keine Priorität hatte“, erklärte mir eine Hausbesitzerin. ${ }^{239}$ Die Bewohner im Viertel 2 bekamen den Anschluss an das städtische Abwassersystem erst ein bis zwei Jahre vor unseren Gesprächen.

Viel Unmut und Frust in konzentrierter Form über die chaotischen Zustände und die Unfähigkeit der Stadtverwaltung, grundlegende Infrastruktur in den neu entstehenden Vierteln zu organisieren, enthält das Protokoll einer großen Einwohnerversammlung von 323 Teilnehmenden mit einem Stadtratsabgeordneten

238 SamOGA, f. 1658, op. 2, d. 109, 1. 17-39.

239 Gespräch mit Familie Salovatov. 
vom 19. August 1958 im Bezirk der ehemaligen Lermontov-Straße, heute Ogahiy. ${ }^{240}$ Die Einwohner kritisieren in recht harschem Ton viele Mängel und Versäumnisse seitens der städtischen Behörden: dass Straßen und Zufahrten fehlten, dass Bewässerungsgräben falsch und nicht vollständig angelegt waren, was Überflutungen bei manchen Häusern verursachte, dass jegliche Einkaufs- und Kinderbetreuungsmöglichkeit fehlte, und schließlich die Korruptheit und Untätigkeit der Kontrollinstanzen. So beschweren sich die Bewohner, dass sie bereits seit zwei Jahren auf die von der Stadt versprochene Wasserleitung und auf die Einrichtung eines Lebensmittelladens warten. Andere klagen über verwirrende und sich teilweise doppelnde Straßenbenennung im Viertel. Mehrere Sprecher nennen korrupte Kontrolleure bzw. Mitarbeiter der städtischen Behörden, die extra Gelder von den Einwohnern sammeln, die Arbeit aber trotzdem nicht erledigen würden. Offenbar waren solche Treffen mit einem Abgeordneten eine Seltenheit, und vermutlich noch seltener standen die Verantwortlichen der jeweiligen Stadtwirtschaftsbereiche Rede und Antwort, da solche Treffen ebenfalls gefordert wurden. ${ }^{241}$

Gleichzeitig wurden die Einwohner für das gute Aussehen der Straße vor ihrer Haustür verantwortlich gemacht. Sie sollten Wege und Gräben reinigen, die Fassaden streichen und für Straßenbeleuchtung sorgen. Herr Salovatov erinnerte sich, dass die Hausbesitzer von der Stadt junge Bäume und anderes Material für Begrünung bekamen und diese in ihrem Viertel entlang der Straße unter Aufsicht pflanzen mussten. Eine große Rolle bei der Organisation von Aktionen wie den subbotniki (große gemeinsame Aufräumarbeiten im Viertel, die traditionsgemäß an Samstagen stattfanden) wurde in aller Regel von einer speziellen Struktur, den so genannten Quartalskomitees oder mahalla-Komitees, übernommen. Dies war ein aus Mitgliedern der Nachbarschaft gewähltes Organ, dessen Befugnisse und Funktionen von einem praktischen Bindeglied zwischen Stadtverwaltung und den im Viertel lebenden Menschen bis hin zu einem Organ der ideologischen Arbeit reichten und das sich im Laufe der Jahre wandelte. ${ }^{242}$ Aus dem Protokoll einer Versammlung des Komitees mit den Einwohnern, die am 9. August 1958 stattfand, wird deutlich, dass das Komitee auch mit dem Einziehen von Steuern beauftragt ${ }^{243}$ und für die Organisation der blagoustroistvo-Arbeiten im Viertel verantwortlich war. ${ }^{244}$

240 SamOGA, f. 26, op. 1, d. 2057, 1. 6-12.

241 SamOGA, f. 26, op. 1, d. 2057, 1. 6-12.

242 Für einen Überblick über die Entwicklung der mahalla-Komitees siehe Abashin 2011.

243 Laut Abashin fiel diese Aufgabe ab dem Jahr 1961 weg.

244 SamOGA, f. 26, op. 1, d. 2057, 1. 40-43. 


\section{Soziale Struktur im chastnyi-Sektor - ein Leben „Nah am Boden“?}

Nach der Schilderung der Bedingungen, vor denen die individuellen Bauherren standen bzw. in die sie hineingezwungen wurden, stellt sich die Frage nach den sozialen Hintergründen und Voraussetzungen, die ein Mensch oder eine Familie erfüllen musste, um sich der Aufgabe eines Hausbaus zu stellen. Mit meiner Forschung kann ich nicht den Anspruch erheben, eine repräsentative Antwort zu geben; die Beobachtungen sowie Fremd- und Selbstbeschreibungen lassen dennoch einige Vermutungen zu.

Die Richtlinien für die Vergabe der Parzellen enthielten eindeutige Hinweise, nach denen Antragsteller aus der Arbeiterklasse, also Mitarbeiter von Fabriken und industriellen Betrieben, zu bevorzugen waren. ${ }^{245}$ Einige der aufgefundenen Zuteilungslisten enthalten auch die Beschäftigungsbezeichnungen der Antragsteller, deren Bandbreite recht groß ist. In einem Beispiel aus dem Jahr 1957 sind in einer Zuteilungsliste 20 Personen aufgeführt, deren Berufe von Hilfsarbeiter und Putz- oder Pflegekraft bis Direktor einer Großmolkerei, Ingenieur und Bibliotheksverwalter reichen. Eine eigene Kategorie, da extra begünstigt ist, bilden in der Regel Kriegsinvalide und Reserveoffiziere. ${ }^{246}$ Die Tätigkeiten, die als einfache Arbeiter- oder Dienstleistungsberufe kategorisiert werden können, scheinen in den mir vorliegenden Beispielen leicht in der Mehrheit zu sein. Auch hatten unter den von mir befragten Hausbesitzern bzw. bei ihren Eltern nur zwei einen akademischen Abschluss; beim Rest handelte es sich um Tischler, Taxifahrer, Fabrikangestellte, Kolchos-Mitglieder oder Buchhalter - alles Berufsgruppen, deren offizielles Einkommen nicht hoch war. So betrug z.B. im Jahr 1968 der Monatslohn eines Buchhalters 80 Rubel pro Monat ${ }^{247}$ und in den Berufen der Kommunalwirtschaft oder im medizinischen Dienst waren es zwischen 70 und 80 Rubel. ${ }^{248}$

Damit wird deutlich, dass Eigenheimbau in Samarkand kein alleiniges Privileg der Eliten bzw. Gruppen aus der höheren Einkommensschicht war, zumindest nicht nach offiziellem Einkommen betrachtet. Es stellt sich aber die Frage, wie die kosten- und arbeitsintensive Aufgabe eines Hausbaus bewältigt werden konnte, wenn man die Preise für das Baumaterial bedenkt und einbezieht, dass

245 Andrusz 1984, S. 72.

246 SamOGA, f. 26, op. 1, d. 1972, 1. 25-26.

247 SamOGA, f. 1617, op. 1, d. 254, 1. 60.

248 Lubin 1984, S. 176. 
die Häuser nicht beliebig (billig), sondern nach einem Typenprojekt gebaut werden sollten und alles zumindest theoretisch einer Kontrolle unterlag.

Anhand der Beobachtungen, die ich im Laufe der Forschung machen konnte, wird deutlich, dass unterschiedliche Voraussetzungen erfüllt sein mussten. Eine davon ist offensichtlich ein gewisses Mindestmaß an handwerklichem Können bzw. entsprechender Begabung. Bereits in der Gesetzgebung zum individuellen Wohnungsbau war vorgesehen, dass zusätzlich zu den Mitteln aus dem Kredit (die offiziell maximal 70 Prozent, in Wirklichkeit aber, wie oben besprochen, offenbar nur ein Viertel der Ausgaben deckten) die Arbeitskraft des Eigenheimbauers und seiner Familie zum Einsatz kommen sollte. Menschen mit handwerklichen Berufen wie Tischler, Schlosser, Bauarbeiter waren hier natürlich im Vorteil. Doch das alleine hätte nicht ausgereicht. Einer der Gesprächspartner, kein Haus-, sondern ein Wohnungseigentümer, kommentierte das Fehlen eines eigenen Hauses in seiner Familie so:

[...] Menschen mit Berufen, die nah am Boden (blizhe k zemle) waren, konnten so was machen [d.h. ein Haus bauen]. Und stell dir vor, ein Lehrer, Dozent oder mein Vater - er kam als Aspirant an das Institut für Karakulschafzucht nach Samarkand, vier Kinder, Mietwohnung in der Altstadt, mit einem Lohn von 100 Rubel - der würde doch so was nicht machen, er hat auf eine Wohnung gewartet, Briefe geschrieben $[. . .]^{249}$

Mit diesem Zitat sprach mein Interviewpartner gleich zwei zentrale Momente an. Zum einen deutet er hier eine wichtige Voraussetzung an, die seinem Vater als nicht-Einheimischem fehlte: ein Netzwerk an Verwandten, Freunden oder Landsleuten, das mit Finanzen und vor allem mit Arbeitskraft oder Kontakten helfen konnte. Der Einsatz der gesamten Familie und/oder der Gemeinschaft wurde von mehreren Gesprächspartnern betont. Die Fähigkeit, diese zu mobilisieren, war zusammen mit einer gewissen Umtriebigkeit von Vorteil bzw. von Nöten, um an Baumaterial und zusätzliches Geld zu gelangen, damit zum Beispiel auch Bauarbeiter bezahlt werden konnten. So hielt eine Familie Schweine, die dann an eine Großfleischerei verkauft wurden. Der Stiefvater einer anderen Interviewpartnerin war Frisör und hatte damit offenbar einen auskömmlichen nicht offiziellen Zuverdienst, sodass „dadurch das Haus fertig gebaut werden konnte“.250

Ein anderer Gesprächspartner - der älteste von allen und der einzige, der noch selbst, nämlich als 16-jähriger, an seinem Haus mitgebaut hatte - war früh bestrebt, den offenbar sehr lukrativen Job eines Taxifahrers zu bekommen, was

249 Gespräch mit A.S.

250 Gespräch mit S. Av. 
ihm zufolge ohne Beziehungen oder Bestechung nicht möglich war. Als er die Stelle letztlich erlangt hatte, konnte er einiges über den offiziellen Lohn hinaus verdienen und seinen Hausbau finanzieren. ${ }^{251}$

Es sind nur wenige Beispiele; sie machen aber deutlich, dass ein Beruf, bei dem Menschen ihr Handwerk entweder direkt für den Bauprozess einsetzen oder als Dienstleistung privat gegen Geld oder andere Leistung anbieten konnten, sehr hilfreich war, um die nötigen Ressourcen für den Hausbau zusammenzutragen. Der zusätzliche Verdienst spielte in der sogenannten second economy, der Schattenwirtschaft des sowjetischen Usbekistans, in der Tat eine große Rolle und überstieg häufig die offiziellen Löhne um ein Vielfaches. ${ }^{252}$

Das zweite Moment, das ich an dem oben angeführten Zitat für wichtig erachte, ist die Metapher, dem Boden nah sein“ (byt‘ blizhe k zemle). Die Bedeutung kann vielfältig interpretiert werden - „bodenständig“ sein, „handwerklich begabt“, auch „alltagstauglich“ oder „überlebensfähig““. 253 Die Metapher erfasst gut das Spektrum der Anforderungen, die ein Hausbau an eine Person bzw. Familie stellte. Die Bodenständigkeit als Metapher, die in dem Zitat als eine Kategorie der sozialen Zugehörigkeit benutzt wird, fügt sich zu der praktischen Bodenständigkeit. Der andere, ähnlich klingende Ausdruck zhit‘ na zemle ,auf dem Boden leben' begegnete mir in unterschiedlichen Formen - sowohl als ein ausdrücklicher Wunsch der Menschen selbst oder als eine kritische Beschreibung von Behördenvertretern die die Popularität dieser Wohnform als „Neigung der Lokalbevölkerung zum Boden“ - tyaga k zemle beschrieben haben. ${ }^{254}$ Der individuelle Hausbau in Samarkand scheint eine Verkörperung von zum Teil artikulierter Haltung zu sein, die von den Behörden auch als solche verstanden wurde und sich im Laufe der Jahre immer wieder in unterschiedlichen Formen durchsetzen konnte.

Eine weitere Rolle beim Bau der Häuser dürfte die Solidarität von Landsleuten unter den Zugewanderten bzw. Deportierten oder bei den bestehenden ethnischen Gruppen gespielt haben. Die Rede ist hier vor allem von drei Gruppen: Armenier, Krimtataren und Eroni in Samarkand. ${ }^{255}$ So tragen (bzw. trugen) einzelne

251 Gespräch mit H.K.

252 Lubin 1984, S. 171-199.

253 Man kann in diesem Spruch durchaus eine wertende Konnotation sehen, doch ich möchte mich hier nicht in die vielen Metaebenen der Bedeutung versteigen.

254 SamOGA f. 1658, op. 2, d. 146, 1. 6-8. Siehe Kapitel 4, die Kritik des Leiters der städtischen Architektur-behörde an der Politik der Landzuteilung.

255 Zur Geschichte der Krimtataren und ihrer Deportation im Zweiten Weltkrieg nach Zentralasien siehe z.B. Williams 2001, S. 374 ff.; über die Armenier von Samarkand siehe Nazaryan 2007; zur Geschichte der Eroni („Iraner“) in Samarkand siehe z.B. Tokhtiev 2012. Zum Überblick über die Minderheiten, die in der Altstadt von Samarkand lebten und leben, siehe Buttino 2015. 
Abschnitte des Großareals von Viertel 1 inoffizielle Toponyme wie „armenische Siedlung“ (armyanskii posyolok) oder ,,iranisches Dorf“ (iran-kishlak). Sie sind offensichtlich nach ihren Einwohnern benannt. Das Leben dieser Gemeinschaften (die heute durch Wegzug ihrer Mitglieder, vor allem im Falle der Krimtataren und Armenier, nicht mehr in dieser Form existieren) stellt ein spannendes, aber eigenes Forschungsthema dar, das hier nur angerissen werden kann. Die Gründe und Mechanismen des Zustandekommens einer solchen kompakten Siedlungsweise waren offenbar je nach Gruppe unterschiedlich.

Die Höfe der Befragten, die sich als Eroni bezeichneten, standen nach Eigenaussage locker in der Gegend verteilt, getrennt durch weitläufige Gärten. Nach dem Abriss bekamen teilweise verwandte Familien eng beieinander liegende Parzellen zugeteilt. Bei Armeniern und Krimtataren gab es das bewusste Bestreben bei der Antragstellung, zusammenhängende Parzellen zu erhalten, was auch den Wünschen der Stadtverwaltung entgegenkam. ${ }^{256}$ Gleichzeitig hatten viele von ihnen handwerkliche Berufen wie Frisör, Schlosser usw. Ein Vertreter der krimtatarischen Minderheit sagte mit Stolz: „Wir Krimtataren sind ja alle Handwerker - wir konnten alles selber machen, Fußboden, Decke, Putz, Ofen, alles, und es hält immer noch.“" ${ }^{257}$

Ob Bauvorhaben als Ersatz für bereits existierende Häuser oder nach einem Antrag - die meisten Bauherren waren schon mit dieser Lebensform vertraut, was ihnen sicherlich half, mit den entstehenden Aufgaben umzugehen. Dazu kam die Bereitschaft, über lange Zeit so unkomfortable Bedingungen in Kauf zu nehmen wie das Wohnen auf einer Baustelle, das Fehlen von Wasserleitung und Kanalisation und den Umgang mit vielen anderen Provisorien. Die herrschende Wohnungsnot ließ gleichzeitig nicht viele Alternativen zu, außer der des Wartens auf eine staatliche Wohnung. Trotz allen Widrigkeiten war die Nachfrage so groß, dass die Stadt sie nicht befriedigen konnte, was Tür und Tor für unkontrollierte Bebauung öffnete.

256 Gespräch mit J.O.

257 Gespräch mit T.T. 